\title{
Guided Algebraic Specification Mining for Failure Simplification
}

\author{
Alexander Elyasov, I.S. Wishnu B. Prasetya, and Jurriaan Hage \\ Dep. of Inf. and Computing Sciences, Utrecht University, Utrecht, The Netherlands \\ $\{$ A.Elyasov, S.W.B.Prasetya, J.Hage $\}$ @uu.nl
}

\begin{abstract}
Software systems often produce logs that capture information about their execution behaviour. When an error occurs, the log file with the error is reported for subsequent analysis. The longer the log file, the harder to identify the cause of the observed error. This problem can be considerably simplified if we reduce the log length, e.g., by removing events which do not contribute towards finding the error. This paper addresses the problem of $\log$ reduction by rewriting the reported log in such a way that it preserves the ability to reproduce the same error. The approach exploits rewrite rules inferred from a set of predefined algebraic rewrite rule patterns, exhibiting such properties as commutativity and identity. The paper presents an algorithm for rewrite rules inference, and a terminating reduction strategy based on these rules. Being log-based the inference algorithm is inherently imprecise. So the inferred rules need to be inspected by a human expert before actually being used for rewriting. The approach is language independent and highly flexible. The paper formally defines all used concepts and discusses a prototype implementation of a log reduction framework. The prototype was empirically validated on a web shop application.
\end{abstract}

Keywords: logging, fault localisation, log reduction, log rewriting, property mining.

\section{Introduction}

Application logs represent an important source of information about the real behavior of a system under test (SUT). A typical application log consists of an alternation of the events emitted by the application and application internal states before or after such events. By means of dynamic inference, often complemented with static analysis, the logs can be transformed into application models, commonly expressed as finite state machines (FSM) [15]. These FSM models are supposed to capture the complex interplay between data and control flow, have a moderate number of states, and at the same time precisely reflect the application semantics. As a recent empirical assessment 14 has shown this task is highly challenging, and in many respects depends on the type of logging abstraction as well as the quality of log data itself. 
On the other hand, when a failure occurs logs, also become valuable for the failure context that helps to track down the root cause of the failure, or at least to reproduce the failure 1311 . Such a failure context consists of the application states and events preceding the failure. In case of failure reproduction, the length of the failure context might directly impede the debugging process. To address this problem several techniques can be employed such as delta-debugging (DD) 27 and FSM model inference 1815. DD searches for a minimal failing sub-sequence that preserves the failure. It runs until no one event can be removed without breaking the ability to produce the failure. In practice, however, DD requires multiple online execution attempts which can be expensive or even not feasible at all. In contrast, once the FSM model of an application is inferred from logs, it can be used for minimisation of the failing sequence. The drawback of dynamically inferred FSM models is that the minimised sequence might lose the possibility to reproduce the failure due to of over-approximation.

To address the restrictions imposed by both DD and FSM inference, we propose a novel approach to failure simplification based on guided mining of algebraic rules and failure revealing test case reduction by rewriting. In comparison with DD, this approach does not require us to replay the failing sequence repeatedly. At the same time, it is less expensive than FSM inference, and it also more strongly resembles the failure related circumstances.

Our approach consists of two phases: 1) inference of rewrite rules from already collected logs based on a set of predefined rewrite rule patterns; and 2) turning the rewrite rules into event rewriting system, which is used for the reduction of the original failing test case to a smaller one that still preserves the failure. The predefined patterns represent common algebraic properties between the application events, such as commutativity and identity. Despite their apparent simplicity, these properties can be successfully used for log reduction, which has been verified on a model web store application example.

The main contributions of this paper are:

- It formally states the log reduction problem.

- A solution is proposed, which exploits the equivalence of event traces with respect to the final states in which these traces can result.

- A terminating and non-increasing reduction strategy is presented.

- Empirical validation is carried out on a web shop application example.

The paper has the following structure. Section 2 introduces a motivating example, the GCD application, and shows how the reduction approach works. In Section 3.1 the formal definitions of execution equivalence and event reduction system are given. The algebraic rewrite rule patterns and the reduction strategy are provided in Section 3.2 and 3.3 respectively. We discuss the implementation of the Log Reduction Framework in Section 4. Results of the empirical validation of inference and reduction parts are presented in Section 5. Related work is considered in Section 6. Section 7 concludes the paper and discusses future work. 


\section{Motivating Example}

In this section, we introduce an example to illustrate the log reduction approach. The example is a GUI application for calculating the greatest common divisor (GCD) of two natural numbers. The application has three text fields $X$ (the first number), $Y$ (the second number), and $R$ (the result), and two buttons calc and clear. When the application is initialised, all fields are empty by default.

An essential part of our approach is to use an event-state logging model. This model is discussed in detail in Section 3 . For now, we only need to know that a $\log$ is composed of the alternation of events and states, where the events are the application events and the states are the abstract states of the application. That is, when an event is triggered, we $\log$ (serialise) the abstract application state at the end of the event, as well as a description of the event itself, which includes the event name (possibly the type) and the values of the event parameters. An event starts its execution in the state where the previous one has finished (except for the first event, which starts in some initial state).

We distinguish the following application events in our GCD example:

\begin{tabular}{l|l} 
Event & \multicolumn{1}{|c}{ Description } \\
\hline set $X(x)$ & the user assigns the value $x$ to the field $X$ \\
set $Y(y)$ & the user assigns the value $y$ to the field $Y$ \\
calc & the user clicks the button calc, which changes the value of $R$ \\
clear & the user clicks the button clear, which erases all fields
\end{tabular}

As an abstract state of the GCD application, let's take the triple $\{x, y, r\}$, where $x, y$ and $r$ are the values of the corresponding fields $X, Y$ and $R$. At the beginning of an execution all fields are empty, consequently the variables associated with them are undefined. If the event calc is called when one of the fields is undefined, then nothing happens. Assume that the application has a fault in the implementation of the event set $X$, namely, $\operatorname{set} X(x)$ always results in assigning zero to the field $X$. The corresponding error will be exposed as a failure in case the calc event is executed from the state where one of the variables $x$ or $y$ is equal to zero.

Now, let us consider the following execution sequence:

$$
\begin{aligned}
& \tau=\{?, ?, ?\} \stackrel{\operatorname{set} X(3)}{\rightarrow}\{0, ?, ?\} \stackrel{\operatorname{set} Y(5)}{\rightarrow}\{0,5, ?\} \stackrel{\text { clear }}{\rightarrow}\{?, ?, ?\} \stackrel{\text { set } X(9)}{\rightarrow}\{0, ?, ?\} \\
& \stackrel{\operatorname{set} Y(4)}{\rightarrow}\{0,4, ?\} \stackrel{\operatorname{set} X(0)}{\rightarrow}\{0,4, ?\} \stackrel{\operatorname{set} Y(3)}{\rightarrow}\{0,3, ?\} \stackrel{\text { set } Y(6)}{\rightarrow}\{0,6, ?\} \stackrel{\text { calc }}{\rightarrow} \text { error }
\end{aligned}
$$

This sequence leads to an error due to an attempt to compute the GCD when $x$ is equal to zero.

For the application model described above, two event sequences are equivalent if they result in the same final state starting from the same initial state. For instance, the following equivalences hold for the GCD application: 


$$
\begin{aligned}
\forall e \in \text { Event }[e \quad ; \operatorname{clear}] & =[\operatorname{clear} \\
{[\operatorname{set} X(x) ; \operatorname{set} Y(y)] } & =[\operatorname{set} Y(y) ; \operatorname{set} X(x)] \\
{\left[\operatorname{set} X(x) ; \operatorname{set} X\left(x^{\prime}\right)\right] } & =\left[\operatorname{set} X\left(x^{\prime}\right)\right. \\
{\left[\operatorname{set} Y(y) ; \operatorname{set} Y\left(y^{\prime}\right)\right] } & =\left[\operatorname{set} Y\left(y^{\prime}\right)\right.
\end{aligned}
$$

The second rule, for instance, says that the order of assigning values to the fields $X$ and $Y$ does not matter with respect to the final state they produce. Applying these rules step by step to the original sequence $\tau$, we can reduce it from nine entries to only four, such that the reduced sequence preserves the original error. Therefore, it gives less than half of the original test case exhibiting the same failure. With this reduced test case it should be easier to discover the error root cause, which consequently may decrease debugging time:

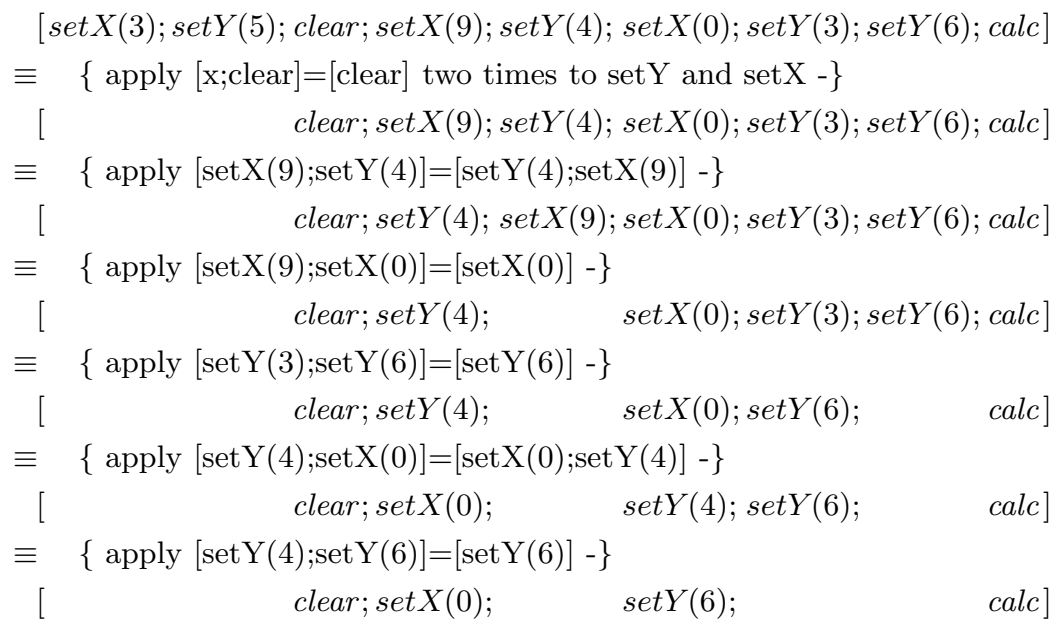

\section{Formal Reduction Theory}

In this section, we formally describe the reduction problem and present our solution. We start by defining an equivalence relation on logs and then describe the logging approach. This equivalence relation lies at the basis of the log reduction, the purpose of which is to decrease the length of a log by rewriting it to a smaller but equivalent one. We propose to learn special rewrite rules from logs that express simple and ubiquitous properties among the application events such as commutativity and idempotence. The learning is effective (Section 5.1), even if the $\operatorname{logs}$ are relatively small (hundreds of entries). Moreover, the application of the rules can result in a significant log reduction (Section 5.2).

\subsection{Log and Event Trace Equivalence}

Following the line of work Lorenzoli et al. [15], we assume that execution traces are produced by an Extended Finite State Machine (EFSM), which implicitly 
underlines the application model. However, in our representation, the EFSM does not contain transition predicates, and the context variables are associated with the states instead of the transitions.

Definition 1 (EFSM). An EFSM $\mathbb{E}$ is a 6-tuple $(S, X, V, E, I, T)$, where

- $S$ is a set of states,

- $X$ is an $n$-dimensional space $X_{1} \times \cdots \times X_{n}$,

- $V$ is a state valuation function on $S$ such that $V: S \rightarrow X$ is injective,

- E is a set of events,

- $P$ is a set of inputs (event parameters),

- $T$ is a transition function such that $T: S \times E \times P \rightarrow S$.

Transition $\left((s, e, p), s^{\prime}\right)$ is denoted as $(s, e, p) \rightarrow s^{\prime}$. For a given $s$ and $e$, if $(s, e, p) \rightarrow s^{\prime}$ for all $p$, we leave out $p$ and write $(s, e) \rightarrow s^{\prime}$. The symbol $\epsilon$ is a special virtual event without parameters that defines the transition $(s, \epsilon) \rightarrow s$. By its definition, an EFSM is deterministic.

Definition 2 (Execution). Given a state $s$ and a sequence of events with parameters $\tau=\left[e_{1}\left(p_{1}\right), \ldots, e_{n}\left(p_{n}\right)\right], \tau$ is an execution of $\mathbb{E}$ starting from the state $s$ if there is a sequence of transitions $\left(s_{1}, e_{1}, p_{1}\right) \rightarrow s_{2},\left(s_{2}, e_{2}, p_{2}\right) \rightarrow$ $s_{3}, \ldots,\left(s_{n}, e_{n}, p_{n}\right) \rightarrow s_{n+1}$ in $\mathbb{E}$ such that $s_{1}=s$.

The execution of $\tau$ starting from $s$ is denoted as $s \mapsto \tau$, and we say that $\tau$ is executable from $s$. The function finalstate returns the final state of the execution $s \mapsto \tau$, that is finalstate $(s \mapsto \tau)=s_{n+1}$.

Definition 3 (Execution Equivalence). Two sequences of events with parameters $\tau_{1}$ and $\tau_{2}$ are equivalent $\left(\tau_{1} \equiv \tau_{2}\right)$ if for all $\left.s \in S: 1\right) \tau_{1}$ is executable from $s\left(s \mapsto \tau_{1}\right)$ if and only if $\tau_{2}$ is executable from $s\left(s \mapsto \tau_{2}\right)$; and 2) finalstate $\left(s \mapsto \tau_{1}\right)=$ finalstate $\left(s \mapsto \tau_{2}\right)$.

Execution equivalence defines an equivalence relation on executions of EFSM. Indeed, it is reflexive, symmetric and transitive by definition. We say that two equivalent executions $\tau_{1} \equiv \tau_{2}$ define a rewrite rule.

Definition 4 (Execution Trace or Log). An execution trace or log $\Sigma$ produced by an execution $s \mapsto \tau$ is the sequence:

$$
\Sigma=\left[\left(\epsilon, x_{1}\right),\left(e_{1}\left(p_{1}\right), x_{2}\right), \ldots,\left(e_{l}\left(p_{n}\right), x_{n+1}\right)\right],
$$

where all states are mapped into their associated values in the domain $X$, that is $x_{i}=V\left(s_{i}\right)$ and $s_{i}$ is an intermediate state of $s \mapsto \tau$.

The function final returns the value corresponding to the final state of the $\log \Sigma$, that is $\operatorname{final}(\Sigma)=x_{n+1}$.

When a user interacts with an application it generates logs in the sense of the definition above. Every state has a uniquely defined element from $X$ associated with it, which is essentially what we see recorded in the log. We call that value 
the application abstract state. Two executions are considered to be equivalent based on those logged values instead of the states. This gives us the following execution equivalence criterion derived from a set of collected logs.

Criterion 1 (Execution Equivalence on Logs). Let $\mathcal{L}$ be a set of logs produced by an application according to the Definition 4. Two event sequences $\tau_{1}$ and $\tau_{2}$ are considered to be equivalent on $\mathcal{L}$ if for all $\Sigma_{1}, \Sigma_{2} \in \mathcal{L}$ resulted from the executions $s \mapsto \tau_{1}$ and $s \mapsto \tau_{2}$ respectively, it implies that final $\left(\Sigma_{1}\right)=\operatorname{final}\left(\Sigma_{2}\right)$.

Definition 5 (Event Reduction System). Given a set of events E, an event reduction system $\Re$ defined by the set of rewrite rules $R$ is a pair $\left(E^{*}, \rightarrow_{R}\right)$, where $E^{*}$ is the set of execution sequences and $\rightarrow_{R}$ is a reduction relation on it.

A reduction step $\tau_{1} \rightarrow_{R} \tau_{2}$ entails the replacement of an occurrence $\pi_{1}$ in $\tau_{1}$ $\left(\tau_{1}=\phi \pi_{1} \psi\right)$ by an equivalent execution $\pi_{2}$, i.e. $\left(\pi_{1} \equiv \pi_{2}\right) \in R$. The result of this reduction is a new execution $\tau_{2}=\phi \pi_{2} \psi$ that is equivalent to $\tau_{1}$ with respect to Definition 3

\subsection{Rewriting Patterns}

As we have already seen in the GCD example, some of the executions are equivalent and can form the bases of the reduction procedure. In general, executions turn out to be equivalent due to the nature of algorithmic languages that contain constructions such as loops, branches and recursions. Some equivalences are application specific, but some others commonly occur in many applications. Below we propose three patterns of the execution equivalences that we call algebraic rewrite rule patterns. They will form the basis for the event reduction in the following. The patterns are quite small (the executions consist of at most two events) and they express common algebraic relations such as commutativity. We believe that algebraic patterns represent commonly occurring equivalences and as a result can often be observed in the logs. That fact increases our confidence of their dynamic inference.

Skip: $[e(p)] \equiv \epsilon$

Obviously, not all events have an effect on the application. Those events that do not interfere with the abstract state at all fall into the category of this pattern, which we call skip-like. The GCD application does not have any skip-like events. However, if the GCD abstract state only consisted of the variables $x$ and $y$, then the calc event would become skip-like with respect to the chosen abstraction.

Zero: $[e(p) ; d(q)] \equiv[d(q)]$

Some events may completely overwrite the effect of some preceding events. We call such events zero-like with respect to those preceding events. In the GCD application, the event clear annuls the effect of set $X$, set $Y$ and calc. A particular case of this pattern is when $e=d$, that is, whatever the application state is, an execution of $e(q)$ always overwrites the effect $e(p)$. The $\operatorname{set} X$ and set $Y$ events are examples of this particular case. 
Com: $[e(p) ; d(q)] \equiv[d(q) ; e(p)]$

The last pattern asserts the property of two events being commutative. For example, the fields $X$ and $Y$ can be updated in any order, and therefore the corresponding events set $X$ and $\operatorname{set} Y$ are commutative, in other words they do not interact with each other.

In the sequel, we only consider event reduction systems formed by rules from these three categories Skip, Zero and Com.

Definition 6 (Algebraic Event Reduction System). We call the event reduction system $\Re_{A}=\left(E^{*}, \rightarrow_{R_{A}}\right)$ algebraic if $R_{A}$ is a disjoint union of $R_{s}, R_{z}$ and $R_{c}$ (denoted by $R_{A}=R_{s} \sqcup R_{z} \sqcup R_{c}$ ), where $R_{s}, R_{z}$ and $R_{c}$ are respective instances of Skip, Zero and Com patterns.

\subsection{Reduction Strategy}

Algorithm 1 presents a reduction procedure for an algebraic event trace reduction system that we later prove to be terminating and non-increasing. It terminates in polynomial time, but does not necessarily produce the maximal possible reduction that is reachable with a given set of rewrite rules. As an input the reduction procedure takes an execution $\tau$ and a set of algebraic rules $R_{A}$ and returns a new execution $\tau^{\prime}$ that is equivalent to $\tau$ but smaller. The procedure consists of the following key steps:

1. The initial set of rules $R_{A}$ is enriched by the procedure EnrichRules. The enrichment consists in overlapping certain categories of rules from $R_{A}$ (this resembles a step of the Knuth-Bendix algorithm [2]). It overlaps Skip and Zero rules (Overlapskip), Zero and Zero rules (OverlapZero), and Zero and Com rules (OverlapMZero). For example, taking the overlap of the rules $a b \rightarrow b$ and $b c \rightarrow c$ and applying them in a different order to the sequence $a b c$, we get $a c \rightarrow c$ as a new possible reduction rule. Crossing Zero and Com rules gives us the mirror zero rules (line 4), which are like zero with respect to reversed event sequence $(b a \equiv b)$. These rules are used later at line 2 to get reduction by means of the ZeroReduce procedure in application to the reversed sequence.

2. The functions GroupZeroCls (GroupMZeroCls) groups Zero (MZero) rules with equal right hand sides in the same equivalence class.

3. Next step is to remove all skip-like events by the procedure ReduceSkip.

4. The last reduction step recursively applies the ZeroReduce procedure to the event trace in both directions (from left to right (line 1) and right to left (line 20) until a fixed point is reached. The procedure ReduceZero looks for the first occurrence of $x$ in $u$. Then it exhaustively applies all rules from $r_{x}$ to the part of $u$ left from $x$ (ReduceImmediateZero at line 5), and it also tries to combine zero with commutative rules (ReduceZeroWithCom at line 7). The latter combination allows us to discover zero reductions that are not enabled by default. 


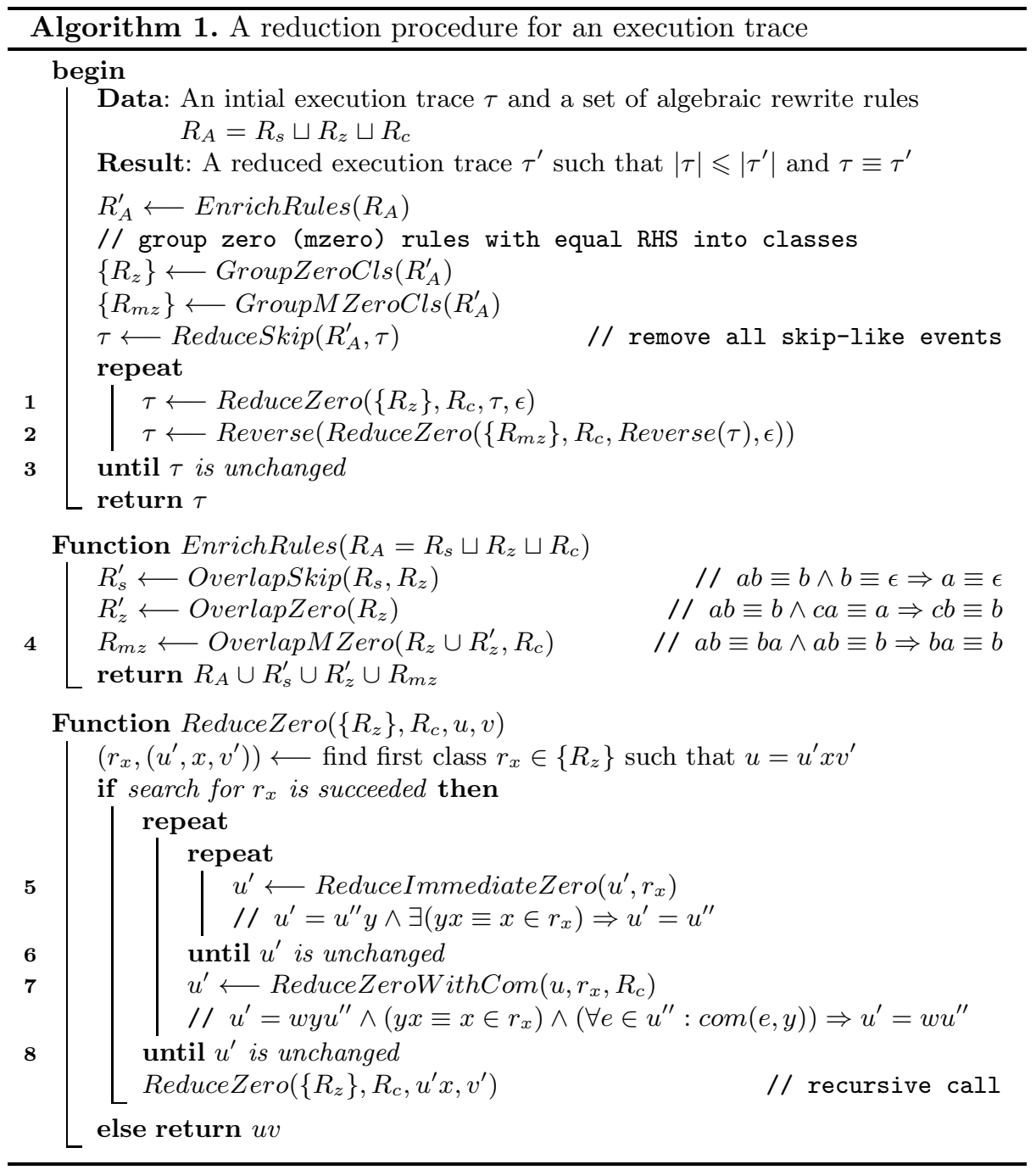

Theorem 1 (Termination). For the algebraic event reduction system $\Re_{A}=\left(E^{*}, \rightarrow_{R_{A}}\right)$, the reduction relation $\rightarrow_{R_{A}}$ implemented by Algorithm 1 is terminating.

Note, that reduction Algorithm 1 essentially applies only Skip and Zero rules, which form a non-cyclic reduction system. In order to prove termination, we need to show that the fix points are guaranteed to be reachable in all three cases (lines 3, 8 and 6). But this fact obviously follows from the invariant that each time the sequence is either reduced or not, the number of rules is finite, and they are acyclic. If the event sequence can not be reduced anymore, we have reached the corresponding fixed point. 
Theorem 2 (Length Reduction). For an algebraic event reduction system $\Re_{A}=\left(E^{*}, \rightarrow_{R_{A}}\right)$, the reduction relation $\rightarrow_{R_{A}}$ implemented by Algorithm 1 is non-increasing.

This property obviously follows the shape of reduction being applied. The algorithm only uses non-increasing reduction rules.

\section{Implementation}

In this section, we describe the architecture of our semi-automated Log Reduction Framework. The framework, including the inference and reduction part, has been implemented in Haskell. The complete structure of the framework is depicted in Figure 1. Given a reliable version App of the application, during the first phase (1. executions) App is subjected to user interaction in order to produce logs. The concrete rewrite rules are inferred (3. concrete rewrite rules) from the set of collected logs (2. collect logs) based on the predefined rewrite rule patterns. After that, they are automatically filtered according to a confidence level (4. check rules) and then passed to the expert (5. report rules to the expert). The rules at this stage might still contain false positives so the human inspection is needed to prevent possible mistakes (6. accept rewrite rules).

At some point a new version $A p p^{\prime}$ of the same application will be realised. It will replace App or will be employed simultaneously with App. We assume that rewrite rules accepted by the expert (phase 6) still hold for $A p p^{\prime}$, otherwise the violation is immediately reported. If the user happens to produce a failure ( 1 '. failure), the log containing the error message (2'. error log) should be passed on subsequent analysis with the purpose to localize the fault and suggest a bug fix. At this point, the second component of our framework goes into action. The failed $\log$ is reduced ( $7 . \log$ rewriting) with the help of the inferred rewrite rules. This log still reproduce the original failure found in $A p p^{\prime}$ but requires less steps. Finally, the reduced $\log$ is inspected (8. reduced $\log$ ) by the tester or developer during the debugging instead of the original one.

\section{Empirical Validation}

The aim of this section is to provide a preliminary evaluation of the Log Reduction Framework. All experiments presented in this section were carried out on an Intel i5 $(2.4 \mathrm{GHz})$ machine with 6GB of RAM under control of Ubuntu 12.04 OS. Reflecting the structure of the framework we provide a separate evaluation of its two main components, Inference and Rewriting. The framework has been deployed for the web shop application flexstore 11. It is an application example for buying of mobile phones, provided by Adobe to demonstrate some features of the Flex SDK. The flexstore has all standard components of a web shop, such as a catalog, product filters and a shopping cart.

${ }^{1}$ http://examples.adobe.com/flex2/inproduct/sdk/flexstore/flexstore.html 


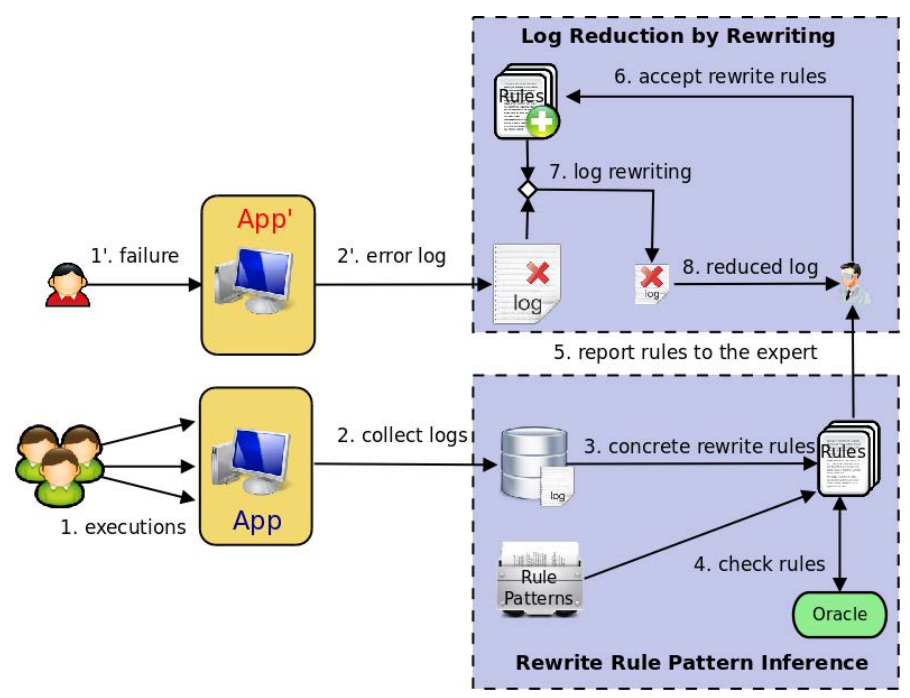

Fig. 1. Log Reduction Framework

We instrumented flexstore GUI events with the help of the FITTEST Automation Framework (AF) [20]. AF allows us to log GUI events and the application abstract states associated with them in the FITTEST Logging Format [20]. The user of AF provides an application abstraction function, which specifies the set of objects and fields to be logged. The resulting log fully conforms to the definition of $\log$ given in Section 3.1 .

\subsection{Inference Results}

We generated a log of length 11000 entries, randomly invoking different flexstore events out of the 23 possible events. We considered two different state projections: high abstraction ( 2 variables, Abs $=$ high) and low abstraction ( 7 variables, Abs = low). False positives were filtered by the use of a confidence level $($ Conf $=$ yes $)$, i.e., all rules with a confidence level lower than 0.99 were not accepted. Without the confidence level $(\mathbf{C o n f}=$ no) it was sufficient for a rule to have at least one positive witness and zero negatives to be accepted as a rewrite rule. As we mentioned already, the inference algorithm can both report false positives and reject false negative rules. Therefore, an expert assessment is required to at least sift out the false positive ones, otherwise, we might end up with a nonequivalent sequence after reduction. We applied the inference algorithm to the log of length 11000, manually verified the results, and then took them as a template to count the number of erroneously accepted and rejected rules in all other measurements during the experiment. The aggregated data of the inference part are shown in Table 1. We used the logs of different sizes (initial segments of length from 100 up to 5000 entries taken from the original log) to infer the rules, and then 
Table 1. Results of pattern inference for flexstore. The events recognised as skip-like are excluded from the subsequent inference of Zero or Com rules.

\begin{tabular}{|c|c|c|c|c|c|c|c|c|c|c|c|c|c|c|c|c|}
\hline \multirow{2}{*}{ Patterns } & \multirow{2}{*}{ Abs } & \multirow{2}{*}{ Conf } & \multirow{2}{*}{$\begin{array}{l}11000 \\
\mathrm{~m} \mathrm{p} \mathrm{n}\end{array}$} & \multicolumn{3}{|c|}{5000} & \multicolumn{3}{|c|}{2500} & \multicolumn{3}{|c|}{1000} & \multicolumn{3}{|c|}{500} & 100 \\
\hline & & & & $\mathrm{m}$ & $p$ & $\mathrm{n}$ & $\mathrm{m}$ & $\mathrm{p}$ & $\mathrm{n}$ & $\mathrm{m}$ & $\mathrm{p}$ & $n$ & $\mathrm{~m}$ & $\mathrm{p}$ & $\mathrm{n}$ & $\mathrm{mp} \mathrm{n}$ \\
\hline \multirow{4}{*}{ Skip } & low & yes & $\begin{array}{|lll|}7 & 0 & 0\end{array}$ & 7 & 0 & 0 & 7 & 0 & 0 & 7 & 0 & 0 & 6 & 0 & 1 & 205 \\
\hline & low & no & $\begin{array}{lll}7 & 0 & 0\end{array}$ & 7 & 0 & 0 & 7 & 0 & 0 & 7 & 0 & 0 & 7 & 0 & 0 & 740 \\
\hline & high & yes & $\begin{array}{lll}9 & 0 & 0\end{array}$ & 9 & 0 & 0 & 9 & 0 & 0 & 9 & 0 & 0 & 8 & 0 & 1 & $\begin{array}{lll}2 & 0 & 7\end{array}$ \\
\hline & high & no & $\begin{array}{lll}9 & 0 & 0\end{array}$ & 9 & 0 & 0 & 9 & 0 & 0 & 9 & 0 & 0 & 9 & 0 & 0 & 950 \\
\hline \multirow{4}{*}{ Zero } & \begin{tabular}{|l|} 
low \\
\end{tabular} & yes & \begin{tabular}{llll|}
26 & 0 & 0
\end{tabular} & 15 & 6 & 11 & 13 & 13 & 13 & & 22 & 15 & 4 & 5 & 22 & $\begin{array}{lll}0 & 0 & 26\end{array}$ \\
\hline & low & no & $\begin{array}{lll}26 & 2 & 0\end{array}$ & 25 & 39 & 1 & 23 & 42 & 3 & 17 & 42 & 9 & & 37 & 14 & 0426 \\
\hline & high & yes & $\begin{array}{lll}9 & 0 & 0\end{array}$ & 1 & 9 & 8 & 1 & 17 & 8 & 0 & 31 & 9 & 0 & 7 & 9 & $\begin{array}{lll}0 & 0 & 9\end{array}$ \\
\hline & high & no & $\begin{array}{lll}26 & 0 & 0 \\
\end{array}$ & 8 & 15 & 1 & 6 & 30 & 3 & 3 & 46 & 6 & 2 & 42 & 7 & $\begin{array}{lll}0 & 4 & 9\end{array}$ \\
\hline \multirow{4}{*}{ Com } & low & yes & \begin{tabular}{lll|l|l|l|l|l|l|l|l} 
& 0 & 0
\end{tabular} & 8 & 3 & 2 & 2 & 0 & 8 & 0 & 0 & 10 & 0 & 1 & 10 & $\begin{array}{llll}0 & 0 & 10\end{array}$ \\
\hline & low & no & $\begin{array}{lll}10 & 0 & 0\end{array}$ & 10 & 5 & 0 & 6 & 8 & 4 & 2 & 3 & 8 & 1 & 1 & 9 & $\begin{array}{lll}0 & 0 & 10\end{array}$ \\
\hline & high & yes & $\begin{array}{lll}7 & 0 & 0\end{array}$ & 6 & 4 & 1 & 1 & 1 & 6 & 0 & 0 & 7 & 0 & 1 & 7 & $\begin{array}{lll}0 & 0 & 7\end{array}$ \\
\hline & high & no & $\begin{array}{lll}7 & 0 & 0\end{array}$ & 7 & 9 & 0 & 4 & 11 & 3 & 1 & 5 & 6 & 0 & 2 & 7 & $\begin{array}{lll}0 & 0 & 7\end{array}$ \\
\hline
\end{tabular}

we compared the inference outcome with the template. As a result, we calculated the number of correctly identified rules ( $m$ column) as well as the number of false positives ( $p$ column) and negatives ( $n$ column). Applying abstraction, we might obtain some new rules as well as lose some old ones. It is clear from Table 1 that to correctly identify all skip rules, it was already sufficient to have 1000 entries in the log. But for Zero or Com rules we missed or wrongly accepted some rules even for the log of 5000 entries. The choice of an appropriate confidence level is always a trade-off between the number of false positive rules we want to avoid and the number of potential false negatives we might lose because of being too exact. As we can see, in case of the Zero and Com rules, there are dozens of candidates, so it is wise to rely on the confidence level to decrease the number of false positives, especially if the amount of data in the logs is limited. For instance, we got rid of 33 false positive rules (reported 6 instead of 39 rules) of the type Zero, learnt from the $\log$ of 5000 entries.

\section{$5.2 \quad$ Reduction Results}

The purpose of the experiments in this subsection is to make an assessment of the failed executions reduction that can be achieved by the framework. We injected faults in the model application flexstore (one fault for each state variable out of seven) and have randomly driven the execution until a failure occurred. For every fault we ran this procedure 100 times in order to deal with randomness of executions. We used the set of concrete rewrite rules inferred from the log of 11000 events in Section 5.1 to form the algebraic event reduction system. The summarized data are presented in Table 2 .

Let us choose a fault and closely consider its characteristics - for example, grand_total fault, which shows the strongest reduction result. The fault here 
Table 2. Failure revealing test case simplification by reduction

max.red.ord, avg.red.ord, min.red.ord are maximum, average and minimum reduction orders respectively; avg.org.len is the average length of the original log, and avg.red.len is the average length of the reduced log.

\begin{tabular}{|l|c|c|c|c|c|}
\hline Failure Type & max.red.ord & avg.red.ord & min.red.ord & avg.org.len & avg.red.len \\
\hline \hline cart_contents & 23 & 5.62 & 1.5 & 35.19 & 6.35 \\
\hline ctlg_contents & 20 & 4.55 & 1.67 & 45.43 & 12.4 \\
\hline comp_contents & 21.2 & 6.07 & 1.33 & 40.42 & 6.44 \\
\hline filter_count & 18 & 4.45 & 1.66 & 45.27 & 12.02 \\
\hline grand_total & 104 & 9.68 & 1.5 & 19.61 & 2.25 \\
\hline \#compare_cart & 34 & 6.82 & 2.72 & 69.11 & 15.75 \\
\hline \#products & 31.6 & 6.83 & 1.99 & 42.82 & 6.59 \\
\hline
\end{tabular}

consist in the restriction imposed on the total price of (variable grand_total) items in the shopping cart. In order to trigger this fault, it is sufficient to open the product catalog, purchase an arbitrary phone and submit the order. The corresponding row in Table 2 indicates that the maximal reduction that is achieved in one out 100 random executions is a factor of 104, although, on average the order of reduction is a factor of 10 . It also says that on average the log is reduced from 20 to just over 2 events.

\section{Related Work}

Since our approach essentially consists of two parts: (1) learning specific rules from logs, and (2) then applying the reduction procedure based on these rules, we accordingly split the related work section.

\subsection{Mining Properties from Logs}

There are various categories of properties that can be learnt from logs. But these properties come under different names: invariants [6], specifications [10] and oracles [21].

The Daikon tool [6] discovers assertions (invariants) that hold at certain program points, e.g., method entry and exit. The assertions are templated predicates over program variables. They express invariants such as constant equality and ordering. Polynomial and array invariants, which Daikon is unable to discover, are presented in [19. DySy obtains invariants that are specific for the observed program executions by using symbolic analysis 3 . The Daikon approach can be extended by the inference of behavioural models of an application [15, which are EFSMs that describe the interplay between data values and component interactions.

Temporal properties have also been thoroughly investigated. Therefore, several tools for learning them have been developed in recent years [71|24]. 
In contrast to Daikon's properties, which represent data-flow dependencies, the temporal properties describe control-flow relations, for instance, the precedence of function calls. The Perracotta tool [24] infers all pairs of events satisfying the property that $p$ must be followed by $s$. A more general class of temporal properties can be inferred by the Ocd tool [7] — a tool for learning and enforcing temporal properties over function and method call sequences. These properties are predefined by templates, which are two-letter regular expressions $\left(a b, a b^{+}\right.$, etc.). Synoptic [1] mines temporal properties, such as $a$ always followed by $b$, and $a$ always precedes by $b$, from partially ordered logs, and then uses these properties to visualise the application model.

The properties proposed in this paper belong to the category of algebraic properties (specifications) 9]. Hankel et al. [10] suggest discovering algebraic specifications by exercising the terms (dynamic part) generated from the algebraic signatures of program classes (static part). Adiheu 8 improves this approach by using Adabu's sequences of legal operations [4] expressed as non-deterministic FSA .

\subsection{Log Reduction}

Reduction always leads to the loss of information, in particular, we sacrifice the completeness of logged data. But there should exist an invariant that continues to hold during the reduction. For instance, someone might be interested in the presence of certain events in the reduced log. The invariant discussed in this paper has the ability to reproduce the same failure as the one contained in the original log.

Wang and Parnas [23] suggest to use trace specifications of software modules as a basis for trace rewriting to simulate module behaviour. The trace specifications completely describe the effect of an event trace execution on the application. In 23] the authors present smart trace rewriting, which is proved to be terminating and confluent. But a formal trace specification is often absent, and its inference brings us back to the questions raised in Section 6.1, namely how to get the specification in the first place.

Clustering techniques are found to be quite useful to reduce the length of logs, in particular, if logs are unstructured. So several clustering algorithms and tools have been introduced, e.g. 16. Clustering assists the reduction in the following ways: 1) similar events can be grouped in clusters so that one event can represent the entire cluster; 2) event correlations can be learnt from logs [28]; and 3 ) outliers (events out the clusters) are potential candidates for anomalies.

Zawawy et al. 26] propose to filter logs with respect to a set of analysis goals and diagnostic hypotheses in order to assist root cause analysis. They suggest two reduction strategies: 1) filter events that are irrelevant to the failure by executing SQL queries generated from annotated goal models 25] capturing application requirements; and 2) apply Latent Semantic Indexing [5] to identify log entries connected with the query representing a particular aspect of the model. Kontogiannis et al. [12] suggest to reduce logs according to the chosen upfront sequence of beacon events. This approach exploits the collection of event 
dependency relations to construct the Event Dependency Graph. The graph is used later on to find all events correlating with the beacon sequence.

Delta Debugging [27] is able to reveal the cause-effect chain of a failure, isolating the relevant variables and values. This chain is essentially the reduced log we are looking for. The method compares the states obtained from a passing and failing run. This requires the ability to replay the failing execution multiple times, which is not required by our approach.

Lee et al. in [13] consider the reduction of log replaying, retaining the ability to reproduce the failure. The reduction is reached by reducing the amount of information that needs to be logged in order to replay the execution. The reduction is carried out at the unit level (loop iterations) by the offline analysis of the enhanced $\log$ - a log resulting from the execution of an instrumented program to collect some axillary runtime information.

BugRedux 11 synthesises and reduces in-house executions that could reproduce the failures observed in the field. BugRedux exploits additional information, for instance call sequences or complete traces, to produce an input that mimics the execution by means of symbolic analysis. Our reduction technique is purely based on the information presented in the log files, and it does not require the application source code to be available.

An FSA is a common way to represent an application model. Such a model can be learnt from logs as in [18 and used for root cause analysis. A failure is then recognised by observing that an execution trace is inconsistent with the FSA. The point of divergence indicates where the abnormal behaviour has started, and the application model can be used to find the shortest path to this point. This gives us a reduced failing execution trace. But, of course, building the precise application model from logs is an expensive task, and a lightweight approach to reduction might be preferable.

\section{Conclusion and Future Work}

In order to discriminate failures that might happen during the execution of an application, programmers try to provide as informative logs as possible. But if a failure occurs, we are not interested in all this excessive information to carry out the root cause analysis of that particular failure.

This paper addresses the issue of failed execution trace simplification that arises if we consider in-house or in the field debugging. We propose to infer algebraic properties among application events, and use them as the basis for our reduction system. We built a prototype of the log reduction framework based on these rules and validated it against the flexstore web application.

Future Work. There are several questions that were left beyond of the scope of this paper and which we consider to be a future work. The reduction algorithm for algebraic log reduction systems presented in this paper, does not guarantee reaching the maximal reduction. We have not yet investigated the desidability of this question. If the answer is positive, it will be interesting to provide an efficient algorithm for maximal reduction. 
Apart from the three rewrite rule patterns discussed in Section 3.2, there exist some other patterns that we did not consider, for instance, $[e(p) ; d(p)] \equiv \epsilon$. This pattern states that $d$ is an inverse of $e$. In general, some practical issues appear if we want to extend our reduction framework with new rewrite rule patterns, namely:

- How to infer the concrete instances of the rewrite rules for this pattern? The inference might be very expensive, require a huge amount of logging data, or generate too many false positives.

- How to incorporate a new pattern into the existing reduction algorithm and to build a new one that is as effective and powerful as the former one?

The answers to these questions require a trade-off between the pattern complexity, inference efficiency and reduction strength. These problems are beyond the scope of this paper and we consider them future work.

In this paper, we do not address the problem of the construction of abstract application state, although the strength of our approach very much relies on the having a good state abstraction. We assume that such abstraction has been already obtained by the use of known techniques [17/22].

Reduction performed with respect to some abstraction might lead to nonexecutable event sequences. Although, it is guaranteed by the approach that they still preserve the failure relevant data, some part of the execution relevant information might be abstracted over. In order to alleviate this issue, reduction can be combined with delta debugging [27, so that it produced both the executable and simplified test case.

Acknowledgements. This work is funded by the EU FITTEST project No. 257574 .

\section{References}

1. Beschastnikh, I., Brun, Y., Schneider, S., Sloan, M., Ernst, M.D.: Leveraging existing instrumentation to automatically infer invariant-constrained models. In: ESEC/FSE, pp. 267-277 (2011)

2. Book, R.V., Otto, F.: String-rewriting systems (1993)

3. Csallner, C., Tillmann, N., Smaragdakis, Y.: DySy: dynamic symbolic execution for invariant inference. In: ICSE, pp. 281-290 (2008)

4. Dallmeier, V., Lindig, C., Wasylkowski, A., Zeller, A.: Mining object behavior with ADABU. In: WODA, pp. 17-24 (2006)

5. Deerwester, S., Dumais, S.T., Furnas, G.W., Landauer, T.K., Harshman, R.: Indexing by latent semantic analysis. Journal of the American Society for Information Science 41 (1990)

6. Ernst, M.D., Perkins, J.H., Guo, P.J., McCamant, S., Pacheco, C., Tschantz, M.S., Xiao, C.: The daikon system for dynamic detection of likely invariants. Sci. Comput. Program., 35-45 (2007)

7. Gabel, M., Su, Z.: Online inference and enforcement of temporal properties. In: ICSE, pp. 15-24 (2010) 
8. Ghezzi, C., Mocci, A., Monga, M.: Efficient recovery of algebraic specifications for stateful components. In: IWPSE, pp. 98-105 (2007)

9. Guttag, J.V., Horning, J.J.: The algebraic specification of abstract data types. Acta Informatica 10, 27-52 (1978)

10. Henkel, J., Diwan, A.: Discovering algebraic specifications from java classes. In: Cardelli, L. (ed.) ECOOP 2003. LNCS, vol. 2743, pp. 431-456. Springer, Heidelberg (2003)

11. Jin, W., Orso, A.: BugRedux: Reproducing Field Failures for In-house Debugging. In: ICSE (2012)

12. Kontogiannis, K., Wasfy, A., Mankovskii, S.: Event clustering for log reduction and run time system understanding. In: SAC, pp. 191-192 (2011)

13. Lee, K.H., Zheng, Y., Sumner, N., Zhang, X.: Toward generating reducible replay logs. In: PLDI, pp. 246-257 (2011)

14. Lo, D., Mariani, L., Santoro, M.: Learning extended FSA from software: An empirical assessment. Journal of Systems and Software 85(9), 2063-2076 (2012)

15. Lorenzoli, D., Mariani, L., Pezzè, M.: Automatic generation of software behavioral models. In: ICSE, pp. 501-510 (2008)

16. Makanju, A., Brooks, S., Zincir-Heywood, A., Milios, E.: LogView: Visualizing event log clusters. In: PST, pp. 99-108 (2008)

17. Marchetto, A., Tonella, P., Ricca, F.: State-based testing of ajax web applications. In: 2008 1st International Conference on Software Testing, Verification, and Validation, pp. 121-130 (2008)

18. Mariani, L., Pastore, F.: Automated identification of failure causes in system logs. In: ISSRE, pp. 117-126 (2008)

19. Nguyen, T., Kapur, D., Weimer, W., Forrest, S.: Using dynamic analysis to discover polynomial and array invariants. In: ICSE, pp. 683-693 (2012)

20. Prasetya, I.S.W.B., Middelkoop, A., Elyasov, A., Hage, J.: D6.1: Fittest logging approach (2011)

21. Shahamiri, S.R., Wan-Kadir, W.M.N., Ibrahim, S., MohdHashim, S.: An automated framework for software test oracle. Information and Software Technology (2011)

22. Tonella, P., Nguyen, C.D., Marchetto, A., Kessler, F.B., Lakhotia, K., Harman, M.: Automated generation of state abstraction functions using data invariant inference (2013)

23. Wang, Y., Parnas, D.L.: Simulating the behavior of software modules by trace rewriting. IEEE Transactions on Software Engineering, 750-759 (1994)

24. Yang, J., Evans, D.: Dynamically inferring temporal properties. In: PASTE, pp. 23-28 (2004)

25. Yu, Y., Wang, Y., Mylopoulos, J., Liaskos, S., Lapouchnian, A., do Prado Leite, J.C.S.: Reverse engineering goal models from legacy code. In: RE, pp. 363-372 (2005)

26. Zawawy, H., Kontogiannis, K., Mylopoulos, J.: Log filtering and interpretation for root cause analysis. In: ICSM, pp. 1-5 (2010)

27. Zeller, A.: Isolating cause-effect chains from computer programs. In: FSE, pp. 1-10 (2002)

28. Zhou, W., Zhan, J., Meng, D., Xu, D., Zhang, Z.: LogMaster: Mining event correlations in logs of large scale cluster systems. CoRR (2010) 